\title{
Diagnostica delle $\beta$-lattamasi a spettro esteso (ESBL) nelle Enterobacteriaceae: problemi e raccomandazioni nella realtà epidemiologica italiana
}

Francesco Luzzaro', Giovanni Gesu², Laura Pagani³, e Gian Maria Rossolini', per conto del Comitato di Studio per gli Antimicrobici (CoSA) dell'Associazione Microbiologi Clinici Italiani 'Laboratorio di Microbiologia, Ospedale di Circolo \& Università dell'Insubria, Varese

'Laboratorio di Microbiologia, Azienda Ospedaliera Niguarda Ca' Granda, Milano

${ }^{3}$ Dipartimento di Scienze Eidologiche, Morfologiche e Cliniche, Sezione di Microbiologia, Università di Pavia, Pavia

${ }^{4}$ Dipartimento di Biologia Molecolare, Sezione di Microbiologia, Università di Siena, Siena

Key words: Enterobacteria, extended-spectrum beta-lactamases, antimicrobial resistance, multi-drug resistance, laboratory methods.

\section{Detection of extended-spectrum B-lactamases (ESBLs) in Enterobacteriaceae: problems and recommendations related to the Italian epidemiology}

\section{INTRODUZIONE}

La produzione di $\beta$-lattamasi è il principale meccanismo di resistenza delle Enterobacteriaceae nei confronti degli antibiotici $\beta$-lattamici, farmaci di grande utilità e di larghissimo impiego per il trattamento delle infezioni causate da enterobatteri.

Oltre 400 differenti $\beta$-lattamasi isolate da numerose specie batteriche sono state descritte in letteratura (13). La classificazione di questi enzimi è complessa, ma può essere riassunta in due schemi principali:

1. la classificazione funzionale di Bush, Jacoby e Medeiros (5), che raggruppa le $\beta$-lattamasi in 4 gruppi funzionali (numerati da 1 a 4 ) e in diversi sottogruppi (indicati con lettere) basandosi sull'attività degli enzimi verso differenti substrati e sulla loro sensibilità agli inibitori (acido clavulanico, sulbactam, tazobactam);

2. la classificazione molecolare di Ambler (1), che raggruppa le $\beta$-lattamasi in 4 classi molecolari (A, B, C e D) basandosi sulla loro struttura primaria.

Varie specie di enterobatteri di interesse clinico producono naturalmente $\beta$-lattamasi a codificazione cromosomica, responsabili di fenotipi di resistenza intrinseca ad alcuni antibiotici $\beta$-lattamici. Inoltre, tutte le principali specie di enterobatteri possono acquisire, per trasferimento genico orizzontale, $\beta$ lattamasi codificate da plasmidi, responsabili di fenotipi di resistenza acquisita ai $\beta$-lattamici.

Klebsiella pneumoniae e Klebsiella oxytoca producono naturalmente enzimi cromosomici di classe A (rispettivamente SHV-1 e K1) che sono in grado di idrolizzare efficientemente le penicilline e le cefalosporine a spettro ristretto. Queste $\beta$-lattamasi, prodotte in genere costitutivamente a basso livello, sono responsabili della resistenza intrinseca che queste due specie mostrano alle penicilline ed alle cefalosporine a spettro ristretto. In alcuni ceppi di $K$. oxytoca può verificarsi una iperproduzione dell'enzima $\mathrm{K} 1$, con fenotipo di resistenza ad alto livello verso l'aztreonam e moderata resistenza al cefotaxime e al ceftriaxone, mentre il ceftazidime rimane attivo (15).

Enterobacter spp., Citrobacter freundii, Morganella morganii, Providencia spp. e Serratia spp. possiedono $\beta$-lattamasi cromosomiche di classe $C$ ( $\beta$-lattamasi di tipo AmpC) attive nei confronti di penicilline, cefalosporine a spettro ristretto, cefamicine e cefalosporine a spettro espanso di terza generazione (es. cefotaxime, ceftriaxone, ceftazidime), e non inibite dall'acido clavulanico e dai solfoni dell'acido penicillinico (sulbactam e tazobactam). La produzione degli enzimi cromosomici di tipo AmpC è di norma inducibile, ma solo le penicilline, le cefalosporine a spettro ristretto e le cefamicine funzionano da induttori: ciò spiega perché queste specie sono intrinsecamente resistenti a quei composti ma non alle cefalosporine di terza generazione. Queste ultime, tuttavia, perdono la loro attività nei confronti di mutanti derepressi che producono costitutivamente l'enzima in grande quantità e che possono essere selezionati durante la terapia con ossimino-cefalosporine. Gli enzimi di tipo AmpC sono scarsamente attivi nei confronti delle cefalosporine a spettro espanso di quarta generazione (es. cefepime, cefpirome) e non sono attivi sui carbapenemi, che mantengono una buona attività anche nei confronti dei ceppi iperproduttori di questi enzimi (15). Alcune specie di enterobatteri, infine, producono $\beta$-lattamasi di tipo AmpC a livelli molto bassi e non inducibili (come Escherichia coli) o non pos- 
siedono $\beta$-lattamasi cromosomiche (come Proteus mirabilis e Salmonella enterica) e sono di norma sensibili anche all'ampicillina. E' soprattutto in queste specie che l'uso di antibiotici $\beta$-lattamici ha favorito la comparsa e la diffusione di $\beta$-lattamasi di classe A ad ampio spettro, mediate da plasmidi, come l'enzima TEM-1. La produzione di questi enzimi determina resistenza acquisita alle penicilline ed alle cefalosporine a spettro ristretto, resistenza che può spesso essere antagonizzata dalla combinazione di tali farmaci con inibitori delle $\beta$-lattamasi (acido clavulanico, sulbactam, tazobactam) (15).

Nei primi anni '80, la resistenza acquisita alle penicilline ed alle cefalosporine a spettro ristretto mediata dalle $\beta$-lattamasi ad ampio spettro (es. TEM-1, SHV-1) è stata efficacemente superata con lo sviluppo e l'introduzione in terapia di nuovi farmaci caratterizzati dalla stabilità a questi enzimi: le cefalosporine di terza generazione (es. cefotaxime, ceftriaxone e ceftazidime). Nel giro di pochi anni, tuttavia, la pressione selettiva esercitata da questi farmaci ha determinato la comparsa di nuove $\beta$-lattamasi a codificazione plasmidica capaci di idrolizzarli, definite come $\beta$-lattamasi a spettro esteso (ESBL).

\section{Definizione di $\beta$-lattamasi a spettro esteso (ESBL)}

Non esiste una definizione universalmente condivisa del termine ESBL. Classicamente, tuttavia, questo termine è stato riservato agli enzimi di classe molecolare A che sono capaci di idrolizzare le ossimino-cefalosporine e i monobattami e che, a differenza delle $\beta$-lattamasi di tipo AmpC, vengono inibiti in vitro dall'acido clavulanico e da altri inibitori delle $\beta$-lattamasi (sulbactam, tazobactam). Le ESBL così definite non idrolizzano i carbapenemi né (a differenza delle $\beta$-lattamasi di tipo AmpC) le cefamicine. Rispetto alle AmpC, le ESBL si differenziano anche per una attività spesso elevata nei confronti delle cefalosporine di quarta generazione quali cefepime e cefpirome (23). In questo articolo sarà fatto riferimento a questa definizione di ESBL.

Oggi sono descritte moltissime ESBL, derivate sia dalle $\beta$-lattamasi ad ampio spettro di tipo TEM e SHV (TEM-1, TEM-2, SHV-1) per poche mutazioni che ne ampliano lo spettro (http://www.lahey.org/studies), sia dal metogenoma microbico esogeno per fenomeni di trasferimento genico orizzontale. Di questo secondo gruppo i principali tipi di ESBL sono rappresentati da CTX-M, PER, GES e VEB. Negli ultimi 10 anni, gli enzimi di tipo CTX-M si sono rapidamente diffusi sia in ambito ospedaliero che in comunità (6). Gli enzimi di tipo VEB, PER e GES sono per lo più di riscontro sporadico ma hanno dimostrato anch'essi la capacità di sostenere episodi epidemici $(9,17,26)$.

A dispetto della loro grande variabilità e della loro differente origine, le ESBL hanno uno spettro di attività simile che comprende le penicilline (eccetto la temocillina), le cefalosporine (ma non le cefamicine, come cefoxitina e cefotetan) e l'aztreonam. I carbapenemi (imipenem, meropenem, ertapenem e doripenem) sono stabili o virtualmente tali verso tutte le ESBL.

La maggior parte delle varianti SHV e alcune delle varianti TEM (ad esempio TEM-3) sono fortemente attive contro cefotaxime e ceftazidime, mentre altre (come TEM-10 e TEM-26) idrolizzano prevalentemente il ceftazidime e sono relativamente meno attive contro cefotaxime e ceftriaxone. Un piccolo numero di esse (ad esempio TEM12) hanno attività di tipo ESBL solo marginale (15). Al contrario, la maggior parte degli enzimi CTX-M idrolizza preferibilmente cefotaxime, ceftriaxone e cefepime piuttosto che il ceftazidime, anche se l'attività ceftazidimasica e la relativa resistenza sono molto aumentate in alcune varianti quali CTX-M-15 (2).

Per definizione, le ESBL di classe A sono sensibili in vitro all'azione dell'acido clavulanico e del tazobactam. In realtà, alcuni ceppi produttori resistono a tutte le combinazioni con gli inibitori oggi in uso. Tale fenotipo può essere dovuto alla iperproduzione di enzima o alla co-produzione di penicillinasi resistenti agli inibitori (per esempio OXA-1). Le classiche $\beta$-lattamasi TEM e SHV possono anche subire mutazioni in modo da diventare resistenti agli inibitori, ma queste modifiche tendono a ridurne l'attività cefalosporinasica. Attualmente nessuno di questi enzimi (né alcuna ESBL di tipo CTX-M, VEB o PER) ha subito variazioni tese a combinare l'attività di tipo ESBL con una significativa resistenza agli inibitori.

\section{Origine, evoluzione e diffusione dei ceppi pro- duttori di ESBL}

Le ESBL di tipo TEM e SHV sono state descritte per la prima volta all'inizio degli anni ' 80 e, ad eccezione del Sud America e del Giappone, sono state le ESBL predominanti fino alla fine degli anni '90 (3). Questi enzimi si sono diffusi prevalentemente in $K$. pneumoniae, in particolare nei reparti di Terapia Intensiva. In questo ambito $\mathrm{i}$ batteri produttori di ESBL hanno causato episodi epidemici clonali di grandi dimensioni e di difficile eradicazione (3). Questo pattern persiste ancora negli USA: i più recenti dati forniti dal National Nosocomial Infections Surveillance (NNIS) System mostrano che, nel 2003, il 5.8\% degli isolati di E. coli ed il $20.6 \%$ degli isolati di 
K. pneumoniae nei reparti di Terapia Intensiva erano produttori di ESBL (21).

Tutti gli enterobatteri possono ospitare geni che codificano per la produzione di ESBL di tipo TEM o SHV, ma le SHV sono più frequenti in Klebsiella, Enterobacter e Serratia, mentre le TEM risultano prevalenti in E. coli, P. mirabilis e Providencia spp. Nel complesso, nessuna singola variante di tipo TEM o SHV è diventata dominante, sebbene SHV-2, SHV-5 e SHV-12 siano comuni in tutto il mondo; TEM-10 e TEM-26 sembrano relativamente frequenti in Nord America (3) mentre TEM-24 e TEM-52/92 sono state spesso trovate in Europa $(23,24)$.

Le ESBL di tipo CTX-M sono state descritte per la prima volta verso la fine degli anni ' 80 in Giappone, Germania ed Argentina (2). Per un lungo periodo questi enzimi sono rimasti rari in Europa mentre si sono rapidamente disseminati in Argentina, dove nel 1990 rappresentavano il 75\% delle ESBL tra gli enterobatteri (2).

Un'evoluzione simile si sta adesso verificando in Europa dove, a partire dal 2000, le CTX-M si sono diffuse in maniera massiccia (soprattutto in E. coli) anche a livello comunitario (16).

Attualmente $E$. coli è il principale produttore di CTX-M, seguito da $K$. pneumoniae e, più raramente, da Salmonella spp., Citrobacter spp., Proteus spp. e M. morganii (16).

Una problematica ancora non abbastanza considerata è la grande diffusione delle ESBL nelle strutture di lungodegenza e nelle residenze sanitarie assistite. Questo aspetto è oggi all'attenzione della comunità medica ma dati epidemiologici in questo contesto sono molto scarsi (23).

\section{Epidemiologia dei batteri produttori di ESBL in Italia}

L'epidemiologia dei batteri produttori di ESBL in Italia è stata investigata in due studi nazionali di sorveglianza eseguiti nel 1999 e nel 2003 (18, 24, 29). I dati ottenuti nei due studi hanno mostrato un moderato aumento della prevalenza di enterobatteri produttori di ESBL nei pazienti ospedalizzati (dal 6.3\% nel 1999 al 7.4\% nel 2003). Nel 2003 è stata osservata anche la presenza di ceppi ESBLpositivi nel 3.5\% dei pazienti ambulatoriali (18).

Le due sorveglianze hanno registrato notevoli differenze riguardo alle specie batteriche prevalenti in ambito ospedaliero nei due periodi considerati. In particolare, la percentuale relativa di $K$. pneumoniae tra tutti i batteri produttori di ESBL è nettamente diminuita (dal 37.1\% del 1999 al 15.1\% del 2003) mentre quella di E. coli è salita dal 10.8 al $31.9 \%$. La presenza di ceppi ESBL-positivi di P. mirabilis (molto frequenti nell'epidemiologia italiana) è rimasta sostanzialmente invariata (per- centuali relative, $25.7 \%$ nel 1999 vs. $26.2 \%$ nel 2003). In aggiunta ai tre classici patogeni, nel 2003 Providencia stuartii (percentuale relativa, $7.1 \%$ ) è emersa in maniera significativa fra $i$ batteri ESBL-positivi. Nei pazienti ambulatoriali, $P$. mirabilis è risultato il più frequente produttore di ESBL (39.2\%), seguito da E. coli (34.2\%) e da $P$. stuartii $(10.1 \%)$.

Notevoli variazioni sono state riscontrate anche in relazione al reparto di provenienza: mentre nello studio del 1999 una alta percentuale dei ceppi era isolata nei reparti di Terapia Intensiva, nel 2003 più del $50 \%$ dei ceppi proveniva dai reparti di Medicina e solo una quota più ridotta dai reparti di Terapia Intensiva. In questo contesto trova anche una spiegazione la ridotta prevalenza di $K$. pneumoniae, patogeno respiratorio particolarmente diffuso in Terapia Intensiva. Nel 1999 più del $50 \%$ degli isolati provenivano da urine mentre le emocolture erano la seconda fonte di isolamento $(16.5 \%)$ seguite dalle ferite chirurgiche $(14.5 \%)$ e dalle basse vie respiratorie $(10.4 \%)$ (29). Nel 2003 la quota di emocolture positive per enterobatteri produttori di ESBL si è ridotta al $6 \%$ (presumibilmente come conseguenza del ridotto isolamento di ceppi produttori di ESBL da pazienti ricoverati in Terapia Intensiva), mentre sono aumentati gli isolamenti da pazienti con infezioni delle vie urinarie (molto frequenti in Medicina Generale). A livello ambulatoriale, più dell' $85 \%$ dei ceppi ESBL-positivi sono stati isolati da pazienti con infezione delle vie urinarie (18).

La sensibilità ai farmaci dei ceppi produttori di ESBL è risultata molto simile nelle due sorveglianze: con l'eccezione di rari isolati di P. mirabilis e di P. stuartii caratterizzati da una sensibilità intermedia all'imipenem, $\mathrm{i}$ carbapenemi sono risultati sempre attivi, mentre la resistenza ad aminoglicosidi e fluorochinoloni era spesso associata alla produzione di ESBL. Nel caso di $K$. pneumoniae, la resistenza associata a gentamicina, amikacina e ciprofloxacina coinvolgeva circa il $50 \%$ dei ceppi (18).

Nelle due sorveglianze sono state analizzate anche le diverse varianti di ESBL presenti. Nel 1999 le varianti di tipo TEM erano le più rappresentate (46.8\%), seguite da SHV (34.6\%) e da ceppi positivi per entrambe le varianti $(11 \%)$, mentre solo una piccola percentuale di enterobatteri $(7.6 \%)$ produceva ESBL di tipo non-TEM e non-SHV. Nel 2003, a soli 4 anni di distanza, la percentuale di ceppi produttori di ESBL nonTEM e non-SHV era salita al $22.3 \%$, rivelando l'emergenza di enzimi di tipo PER (2.6\%) e soprattutto l'ampia diffusione di enzimi della famiglia CTX-M (19.7\%) (18). Le CTX-M erano 
diffuse in tutti i centri partecipanti, risultando molto frequenti in $E$. coli (con punte dell' $80 \%$ di prevalenza) e in minor misura in K. pneumoniae. L'analisi molecolare ha dimostrato una elevata prevalenza della variante CTX-M-1, seguita dalle varianti CTX-M-15 e CTX-M-32 (varianti con aumentata attività ceftazidimasica appartenenti alla stessa linea evolutiva) (20). In linea con questa osservazione, uno studio effettuato su ceppi di E. coli produttori di ESBL conservati presso il Laboratorio di Microbiologia dell'Ospedale di Varese ha evidenziato che i ceppi produttori di CTX-M sono comparsi alla fine del 1999 e che la loro frequenza di isolamento è progressivamente aumentata fino ad arrivare nel 2003 al 38\% di tutti i ceppi ESBL-positivi (4).

Per quanto riguarda l'enzima PER-1, identificato per la prima volta nel 1995 in Turchia in Pseudomonas aeruginosa e successivamente anche in Italia alla fine degli anni '90 (17), la sorveglianza del 2003 ha dimostrato che questo enzima si è diffuso anche in $P$. mirabilis, $P$. stuartii e Providencia rettgeri (25).

Aspetti diagnostici della produzione di ESBL negli enterobatteri

Perché è importante diagnosticare correttamente i ceppi produttori di ESBL

La diagnostica della produzione di ESBL nelle Enterobacteriaceae rappresenta un aspetto importante nell'attività dei laboratori di Microbiologia Clinica per le implicazioni che riveste ai fini dell'orientamento terapeutico. Quando la produzione di ESBL determina valori di MIC per le cefalosporine a spettro espanso e per i monobattami superiori al breakpoint clinico di resistenza, l'interpretazione del risultato è inequivocabile. La produzione di ESBL, tuttavia, non sempre determina valori di MIC superiori al breakpoint clinico per la resistenza, in relazione alla diversa attività idrolitica delle differenti varianti enzimatiche, alla quantità di enzima prodotto ed alla variabile capacità degli antibiotici $\beta$-lattamici di attraversare la membrana esterna. In questi casi, nonostante l'apparente sensibilità in vitro, le cefalosporine a spettro espanso possono fallire nel trattare le infezioni causate da batteri ESBL-positivi $(22,27)$. Sulla base di tali considerazioni vi è un ampio consenso a livello internazionale per interpretare in via cautelativa tutti i ceppi produttori di ESBL come clinicamente resistenti a penicilline, cefalosporine (escluse le cefamicine) e monobattami (7).

In questo contesto, la valutazione microbiologica della produzione di ESBL assume una fondamentale importanza per una corretta interpretazione e refertazione dei risultati dell'antibiogramma.

\section{Come diagnosticare la produzione di ESBL}

La rilevazione delle ESBL prevede 2 passaggi successivi:

- un test di screening per identificare gli isolati potenzialmente produttori di ESBL;

- un test di conferma da effettuare sui ceppi risultati positivi al test di screening (questo test è mirato a distinguere gli isolati produttori di ESBL da quelli resistenti alle cefalosporine sulla base di meccanismi diversi, quali l'iperproduzione di $\beta$-lattamasi cromosomiche e/o la riduzione di permeabilità della membrana esterna).

\section{1) Test di screening}

I test di screening consistono nel saggiare la sensibilità a vari $\beta$-lattamici utilizzando breakpoint modificati con farmaci utilizzati come indicatori. Il Clinical and Laboratory Standards Institute (CLSI) suggerisce di testare come indicatori cefotaxime, ceftazidime, ceftriaxone, cefpodoxime ed aztreonam (7). In Tabella 1 sono riportati i diametri e le MIC considerati dal CLSI come indicativi di sospetta produzione di ESBL per E. coli, Klebsiella spp. e P. mirabilis (7). La ridotta sensibilità anche verso uno solo dei farmaci saggiati come indicatori suggerisce la sospetta produzione di ESBL. Poiché le diverse ESBL possono avere una diversa attività idrolitica nei confronti delle diverse molecole, non esiste un singolo farmaco indicatore capace di rilevare la totalità degli isolati produttori di ESBL e, in linea di principio, più farmaci si saggiano e maggiore è la sensibilità di rilevazione. Il cefpodoxime è considerato generalmente il miglior compromesso come farmaco indicatore unico, ma può fallire nella rilevazione di isolati che producono enzimi ad attività strettamente ceftazidimasica (ad esempio TEM-10, TEM-12, TEM-26). Poiché esiste tale possibilità, è consigliabile utilizzare almeno due molecole come indicatori: il ceftazidime (un buon substrato per le varianti TEM e SHV, incluse le ceftazidimasi più strette) ed il cefotaxime (che è un buon substrato per tutte le ESBL di tipo CTX-M). Mentre il ceftazidime è una molecola essenziale per rivelare la presenza di batteri ESBL-positivi a prevalente attività ceftazidimasica, il cefotaxime può essere adeguatamente sostituito (come indicatore) dal ceftriaxone o dal cefpodoxime che hanno dimostrato caratteristiche simili a quella del cefotaxime ai fini di rivelare la produzione di enzimi ad attività prevalentemente cefotaximasica, incluso l'enzima CTX-M-1, ampiamente diffuso in Italia (20).

Per quanto riguarda le specie batteriche diverse da 
Tabella I: Test di screening per la produzione di ESBL in Klebsiella pneumoniae, Klebsiella oxytoca, Escherichia coli e Proteus mirabilis (7)

\begin{tabular}{|c|c|c|c|}
\hline Metodo & \multicolumn{2}{|c|}{ Diffusione in agar } & Diluizione in brodo \\
\hline Terreno & \multicolumn{2}{|c|}{ Agar Mueller-Hinton } & $\begin{array}{c}\text { Brodo Mueller-Hinton con } \\
\text { aggiunta di cationi }\end{array}$ \\
\hline Inoculo & \multicolumn{2}{|c|}{0.5 McFarland } & 0.5 McFarland \\
\hline \multicolumn{4}{|c|}{ Per K. pneumoniae, K. oxytoca, E. coli } \\
\hline \multirow{11}{*}{$\begin{array}{l}\text { Concentrazione } \\
\text { di antibiotico }\end{array}$} & Cefpodoxime & $10 \mu g$ & $4 \mu g / \mathrm{ml}$ \\
\hline & Ceftazidime & $30 \mu \mathrm{g}$ & $\mathrm{l} \mu \mathrm{g} / \mathrm{ml}$ \\
\hline & Aztreonam & $30 \mu g$ & $\mathrm{l} \mu \mathrm{g} / \mathrm{ml}$ \\
\hline & Cefotaxime & $30 \mu g$ & $\mathrm{l} \mu \mathrm{g} / \mathrm{ml}$ \\
\hline & Ceftriaxone & $30 \mu \mathrm{g}$ & $\mathrm{I} \mu \mathrm{g} / \mathrm{ml}$ \\
\hline & \multicolumn{2}{|c|}{$\begin{array}{c}\text { Diametri che indicano la } \\
\text { possibile produzione di ESBL }\end{array}$} & $\begin{array}{c}\text { MIC che indicano la } \\
\text { possibile produzione di ESBL }\end{array}$ \\
\hline & Cefpodoxime & $\leq 17 \mathrm{~mm}$ & $\geq 8 \mu \mathrm{g} / \mathrm{ml}$ \\
\hline & Ceftazidime & $\leq 22 \mathrm{~mm}$ & $\geq 2 \mu \mathrm{g} / \mathrm{ml}$ \\
\hline & Aztreonam & $\leq 27 \mathrm{~mm}$ & $\geq 2 \mu \mathrm{g} / \mathrm{ml}$ \\
\hline & Cefotaxime & $\leq 27 \mathrm{~mm}$ & $\geq 2 \mu \mathrm{g} / \mathrm{ml}$ \\
\hline & Ceftriaxone & $\leq 25 \mathrm{~mm}$ & $\geq 2 \mu \mathrm{g} / \mathrm{ml}$ \\
\hline \multicolumn{4}{|l|}{ Per P. mirabilis } \\
\hline \multirow{7}{*}{$\begin{array}{l}\text { Concentrazione } \\
\text { di antibiotico }\end{array}$} & Cefpodoxime & $30 \mu g$ & $\mathrm{l} \mu \mathrm{g} / \mathrm{ml}$ \\
\hline & Ceftazidime & $30 \mu \mathrm{g}$ & $\mathrm{l} \mu \mathrm{g} / \mathrm{ml}$ \\
\hline & Cefotaxime & $30 \mu g$ & $\mathrm{l} \mu g / \mathrm{ml}$ \\
\hline & \multicolumn{2}{|c|}{$\begin{array}{l}\text { Diametri che indicano la } \\
\text { possibile produzione di ESBL }\end{array}$} & $\begin{array}{c}\text { MIC che indicano la } \\
\text { possibile produzione di ESBL }\end{array}$ \\
\hline & Cefpodoxime & $\leq 22 \mathrm{~mm}$ & $\geq 2 \mu \mathrm{g} / \mathrm{ml}$ \\
\hline & Ceftazidime & $\leq 22 \mathrm{~mm}$ & $\geq 2 \mu \mathrm{g} / \mathrm{ml}$ \\
\hline & Cefotaxime & $\leq 27 \mathrm{~mm}$ & $\geq 2 \mu \mathrm{g} / \mathrm{ml}$ \\
\hline
\end{tabular}

E. coli, Klebsiella spp. e P. mirabilis, non esistono criteri codificati dal CLSI per sospettare la produzione di ESBL. A nostro parere, tuttavia, in linea con le raccomandazioni di altre Società scientifiche (11), sembra adeguato utilizzare gli stessi criteri anche per le altre specie di enterobatteri.

\section{2) Test di conferma}

La conferma della produzione di ESBL può essere effettuata mediante test fenotipici basati sulla dimostrazione della sinergia tra ossimino-cefalosporine e acido clavulanico. Tali metodi includono il test del doppio disco ed il test di combinazione.

\section{a) Test del doppio disco}

Il test di approssimazione mediante doppio disco, proposto da Jarlier alla fine degli anni '80 (14), si esegue come un classico test di diffusione in agar in cui dischi di cefotaxime, ceftazidime, aztreonam e ceftriaxone $(30 \mu \mathrm{g})$ sono posizionati intorno ad un disco di amoxicillina-clavulanato $(20+$ $10 \mu \mathrm{g}$ ) posto al centro della piastra. La distanza centro-centro fra i dischi di $\beta$-lattamici e il disco con l'inibitore, originariamente proposta a 30 $\mathrm{mm}$, può essere aumentata fino a $40 \mathrm{~mm}$ oppure ridotta a $20-25 \mathrm{~mm}$ in funzione dell'attività dell'enzima e della specie saggiata. Il test è positivo quando compare una distorsione della zona che circonda il $\beta$-lattamico in prossimità del disco di amoxicillina-clavulanato. Questo effetto si manifesta in genere come un allargamento dell'alone di inibizione (immagine a tappo di spumante). Il test di approssimazione usa dischi disponibili in commercio ed è di facile esecuzione, ma il fatto che una chiara immagine di sinergia dipenda dalla distanza tra i dischetti lo rende difficilmente standardizzabile. Aspetti caratteristici di test positivi relativi alla produzione delle più comuni ESBL circolanti in Italia sono mostrati in Figura I $(K$. pneumoniae, SHV-12), Figura II (E. coli, CTXM-1) e Figura III (P. mirabilis, TEM-92).

\section{b) Test di combinazione}

Il test di combinazione consiste nel saggiare un $\beta$ lattamico da solo ed in presenza di un inibitore delle $\beta$-lattamasi, in modo da valutare il recupero dell'attività del $\beta$-lattamico in presenza dell'inibitore. Il test può essere eseguito col metodo della diffusione da disco o con metodi che determinano la MIC.

Per quanto riguarda la diffusione da disco, il CLSI raccomanda di eseguire il test seconda la metodica standard di diffusione in agar e considerare significativo per la produzione di ESBL un aumento $\geq 5$ $\mathrm{mm}$ del diametro dell'alone di inibizione per ciascuno degli antibiotici $\beta$-lattamici testati in combinazione con acido clavulanico rispetto al $\beta$-lattamico saggiato da solo (Tabella II).

Riguardo ai metodi di combinazione basati sulla determinazione delle MIC, il CLSI raccomanda di utilizzare un test standard di diluizione in brodo e di considerare il risultato significativo per la produzione di ESBL quando la MIC del $\beta$-lattamico in presenza di acido clavulanico (a concentrazione costante di $4 \mu \mathrm{g} / \mathrm{ml}$ ) risulta ridotta di almeno 3 diluizioni al raddoppio rispetto alla MIC del $\beta$-lattamico saggiato da solo (Tabella II).

Il metodo di combinazione ha il vantaggio di essere standardizzato ed è quello raccomandato dal CLSI per confermare la produzione di ESBL

Tabella II: Test di conferma per la produzione di ESBL in Klebsiella pneumoniae, Klebsiella oxytoca, Escherichia coli e Proteus mirabilis (7)

\begin{tabular}{|c|c|c|c|}
\hline Metodo & \multicolumn{2}{|c|}{ Diffusione in agar } & Diluizione in brodo \\
\hline Terreno & \multicolumn{2}{|c|}{ Agar Mueller-Hinton } & $\begin{array}{l}\text { Brodo Mueller-Hinton } \\
\text { con aggiunta di cationi }\end{array}$ \\
\hline Inoculo & \multicolumn{2}{|c|}{0.5 McFarland } & 0.5 McFarland \\
\hline $\begin{array}{l}\text { Concentrazione } \\
\text { di antibiotico }\end{array}$ & $\begin{array}{l}\text { Ceftazidime } \\
\text { Ceftazidime }+ \text { acido } \\
\text { clavulanico } \\
\text { Cefotaxime } \\
\text { Cefotaxime + acido } \\
\text { clavulanico }\end{array}$ & $\begin{array}{l}30 \mu \mathrm{g} \\
30 / 10 \mu \mathrm{g} \\
30 \mu \mathrm{g} \\
30 / 10 \mu \mathrm{g}\end{array}$ & $\begin{array}{c}0.25-128 \mu \mathrm{g} / \mathrm{ml} \\
0.25 / 4-128 / 4 \mu \mathrm{g} / \mathrm{ml} \\
0.25-64 \mu \mathrm{g} / \mathrm{ml} \\
0.25 / 4-64 / 4 \mu \mathrm{g} / \mathrm{ml}\end{array}$ \\
\hline $\begin{array}{l}\text { Interpretazione } \\
\text { del risultato }\end{array}$ & $\begin{array}{l}\text { Un incremento del di } \\
\text { di uno dei due antibic } \\
\text { combinazione con ac } \\
\text { rispetto alle molecole }\end{array}$ & $\begin{array}{l}\text { tro } \geq 5 \mathrm{~mm} \\
\text { saggiati in } \\
\text { clavulanico } \\
\text { gole = ESBL }\end{array}$ & $\begin{array}{l}\text { Una riduzione della MIC } \\
\geq 3 \text { diluizioni di uno dei } \\
\text { due antibiotici saggiati in } \\
\text { combinazione con l'acido } \\
\text { clavulanico rispetto alle } \\
\text { molecole singole = ESBL }\end{array}$ \\
\hline
\end{tabular}




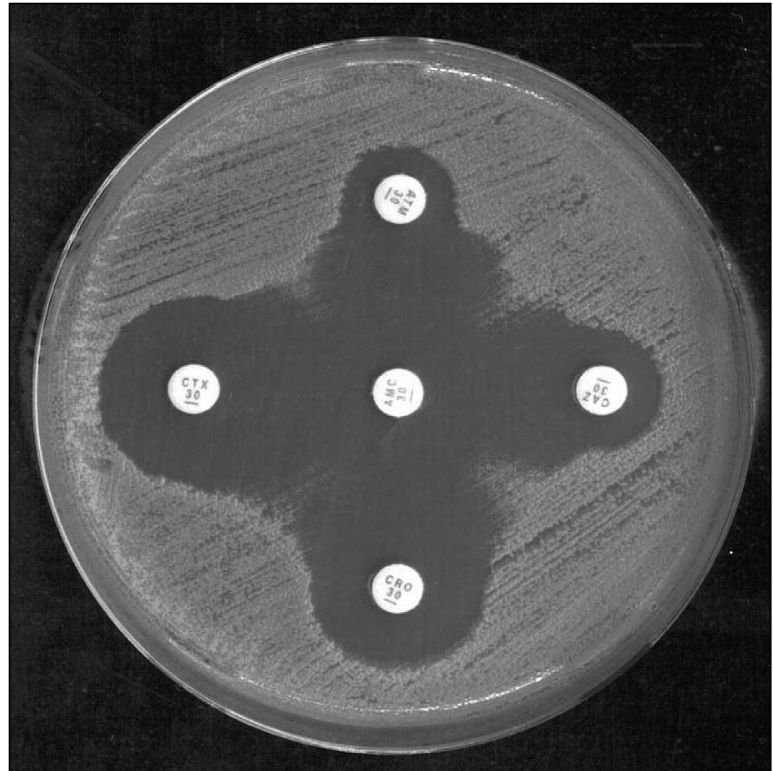

Figura I. Conferma della produzione di ESBL mediante test del doppio disco in Klebsiella pneumoniae produttore di SHV-I2. In alto: ATM, aztreonam $(30 \mu \mathrm{g} / \mathrm{ml})$.

In basso: CRO, ceftriaxone $(30 \mu \mathrm{g} / \mathrm{ml})$.

A sinistra: CTX, cefotaxime $(30 \mu \mathrm{g} / \mathrm{ml})$.

A destra: CAZ, ceftazidime $(30 \mu \mathrm{g} / \mathrm{ml})$.

Al centro: AMC, amoxicillina + acido clavulanico $(20+10$ $\mu g / \mathrm{ml})$.

Distanza centro-centro $25 \mathrm{~mm}$.

in E. coli, Klebsiella spp. e P. mirabilis. Per garantire la massima sensibilità del test, il CLSI suggerisce di saggiare sia il ceftazidime che il cefotaxime, da soli ed in combinazione con acido clavulanico (7). Infatti, con enzimi ad attività strettamente cefotaximasica (ad esempio CTXM-1) il test può risultare positivo solo utilizzando il cefotaxime, mentre con enzimi ad attività strettamente ceftazidimasica (ad esempio TEM-26) il test può risultare positivo solo utilizzando il ceftazidime. L'aspetto dei test di combinazione ottenuti mediante diffusione da disco nei casi menzionati è riportato in Figura IV (E. coli, CTX-M$1)$ e in Figura V (P. stuartii, TEM-26).

Una alternativa ampiamente diffusa nei laboratori di Microbiologia Clinica consiste nell'uso dell'Etest ESBL (AB Biodisk). Questo metodo utilizza strisce che forniscono allo stesso tempo la MIC di una cefalosporina (ceftazidime o cefotaxime) da sola ed in combinazione con acido clavulanico. Anche nel caso dell'Etest, la produzione di ESBL è confermata se l'acido clavulanico riduce la MIC della cefalosporina di almeno 3 diluizioni al raddoppio. In Figura VI è riportato l'aspetto di un test positivo per K. pneumoniae produttore di SHV-12.

Complessivamente, il metodo di combinazione mediante diffusione in agar è un buon compromesso fra costi e sensibilità ma richiede una parti-

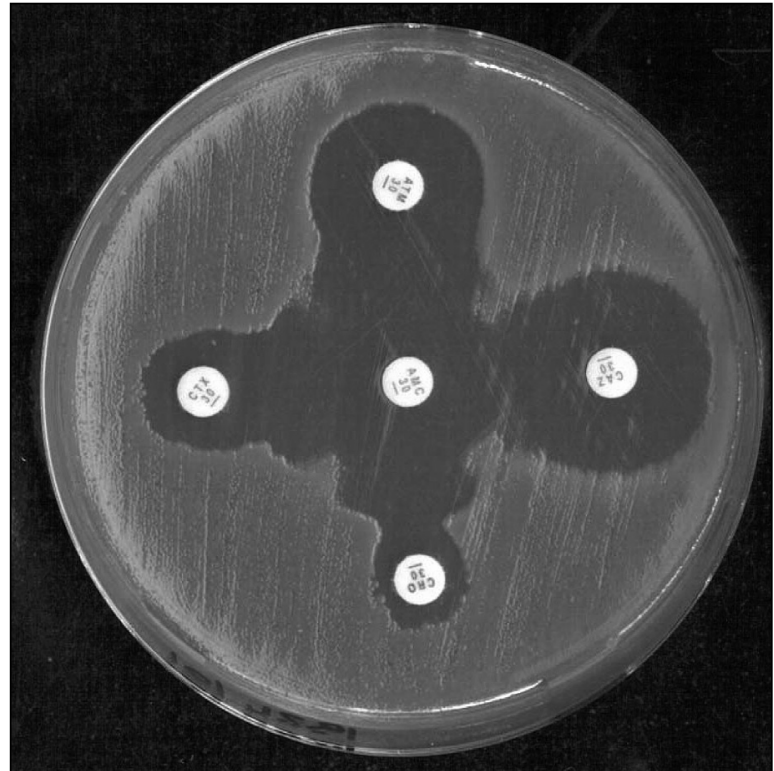

Figura II. Conferma della produzione di ESBL mediante test del doppio disco in Escherichia coli produttore di CTX-M-I.

In alto: ATM, aztreonam $(30 \mu \mathrm{g} / \mathrm{ml})$.

In basso: CRO, ceftriaxone $(30 \mu \mathrm{g} / \mathrm{ml})$.

A sinistra: CTX, cefotaxime $(30 \mu \mathrm{g} / \mathrm{ml})$.

A destra: $C A Z$, ceftazidime $(30 \mu \mathrm{g} / \mathrm{ml})$.

Al centro: AMC, amoxicillina + acido clavulanico $(20+10$ $\mu g / m l)$.

Distanza centro-centro $25 \mathrm{~mm}$.

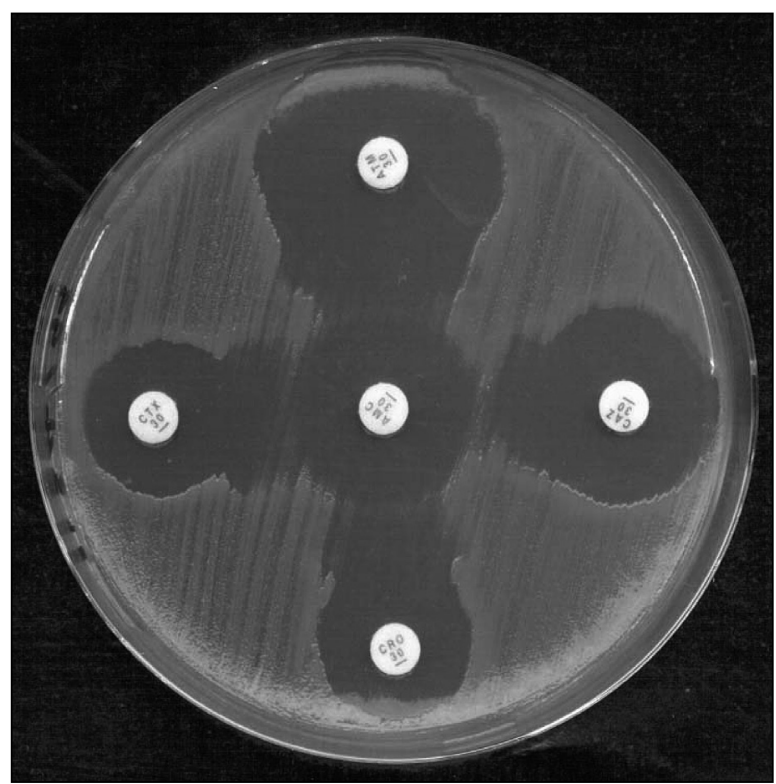

Figura III. Conferma della produzione di ESBL mediante test del doppio disco in Proteus mirabilis produttore di TEM-92. In alto: ATM, aztreonam $(30 \mu \mathrm{g} / \mathrm{ml})$.

In basso: CRO, ceftriaxone $(30 \mu \mathrm{g} / \mathrm{ml})$.

A sinistra: CTX, cefotaxime $(30 \mu \mathrm{g} / \mathrm{ml})$.

A destra: CAZ, ceftazidime $(30 \mu \mathrm{g} / \mathrm{ml})$.

Al centro: AMC, amoxicillina + acido clavulanico $(20+10$ $\mu \mathrm{g} / \mathrm{ml})$.

Distanza centro-centro $30 \mathrm{~mm}$ 


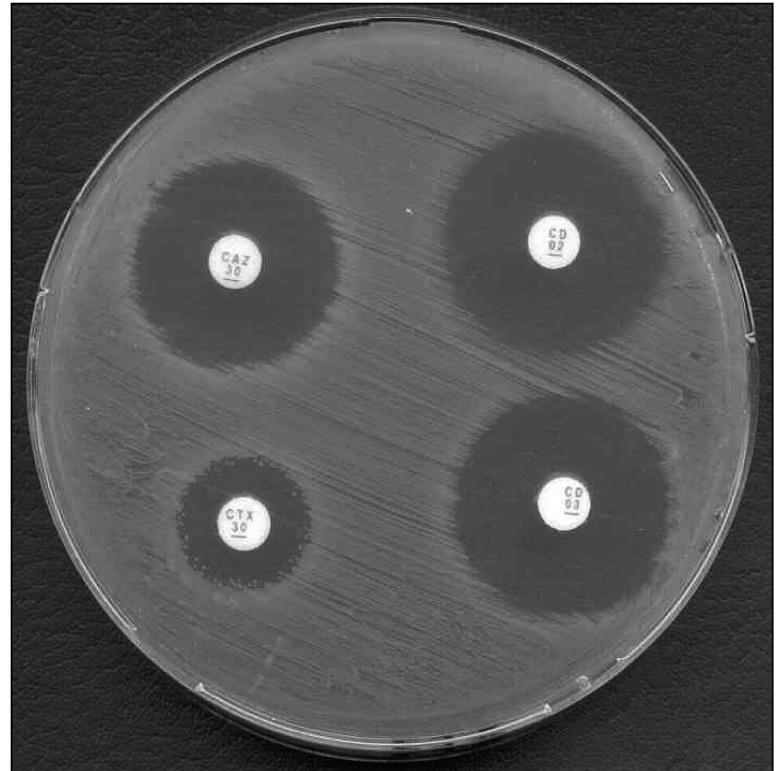

Figura IV. Conferma della produzione di ESBL mediante test di combinazione (diffusione da disco) in Escherichia coli produttore di CTX-M-I.

In alto a sinistra: CAZ, ceftazidime (alone di inibizione, $26 \mathrm{~mm}$ ). In alto a destra: CD02, ceftazidime + acido clavulanico (alone di inibizione, $28 \mathrm{~mm}$ ).

In basso a sinistra: CTX, cefotaxime (alone di inibizione, $14 \mathrm{~mm}$ ). In basso a destra: CD03, cefotaxime + acido clavulanico (alone di inibizione, $28 \mathrm{~mm}$ ).

colare attenzione nella valutazione del risultato quando le differenze tra gli aloni sono vicine al limite soglia (tra 4 e $6 \mathrm{~mm}$ ). Questi problemi non sono inattesi visto che gli standard di produzione dei dischi permettono fino al $50 \%$ di tolleranza nel contenuto dei farmaci. Per questa ragione, il test di combinazione deve sempre essere eseguito anche su un ceppo di controllo ESBL-negativo. Nel test di controllo negativo, gli aloni di inibizione della cefalosporina da sola ed in combinazione con acido clavulanico dovrebbero essere uguali o con una differenza massima di $2 \mathrm{~mm}$ : una differenza maggiore indica un deterioramento dei dischi o comunque un malfunzionamento del test.

c) Controllo di qualità

Per il controllo di qualità, il CLSI raccomanda di utilizzare come controllo positivo il ceppo di $K$. pneumoniae ATCC 700603 (che produce l'enzima SHV-18), mentre la Health Protection Agency e la British Society for Antimicrobial Chemotherapy raccomandano ceppi di $E$. coli con enzimi TEM-3, TEM-10 e CTX-M-3 (rispettivamente disponibili come NCTC 13351, 13352 e 13353). I ceppi di E. coli ATCC 25922 o NCTC 10418 possono essere utilizzati come controlli negativi.

d) Problemi particolari con i test di conferma

Come per il test di screening, il CLSI prevede l'e-

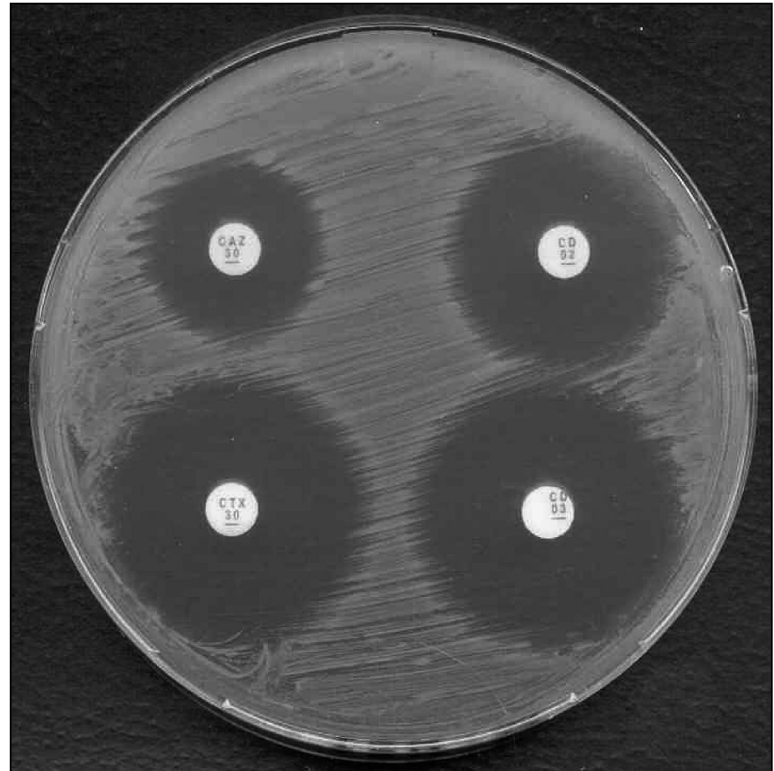

Figura V. Conferma della produzione di ESBL mediante test di combinazione (diffusione da disco) in Providencia stuartii produttore diTEM-26.

In alto a sinistra: CAZ, ceftazidime (alone di inibizione, $22 \mathrm{~mm}$ ). In alto a destra: CD02, ceftazidime + acido clavulanico (alone di inibizione, $30 \mathrm{~mm}$ ).

In basso a sinistra: CTX, cefotaxime (alone di inibizione, $30 \mathrm{~mm}$ ). In basso a destra: CD03, cefotaxime + acido clavulanico (alone di inibizione, $32 \mathrm{~mm}$ ).

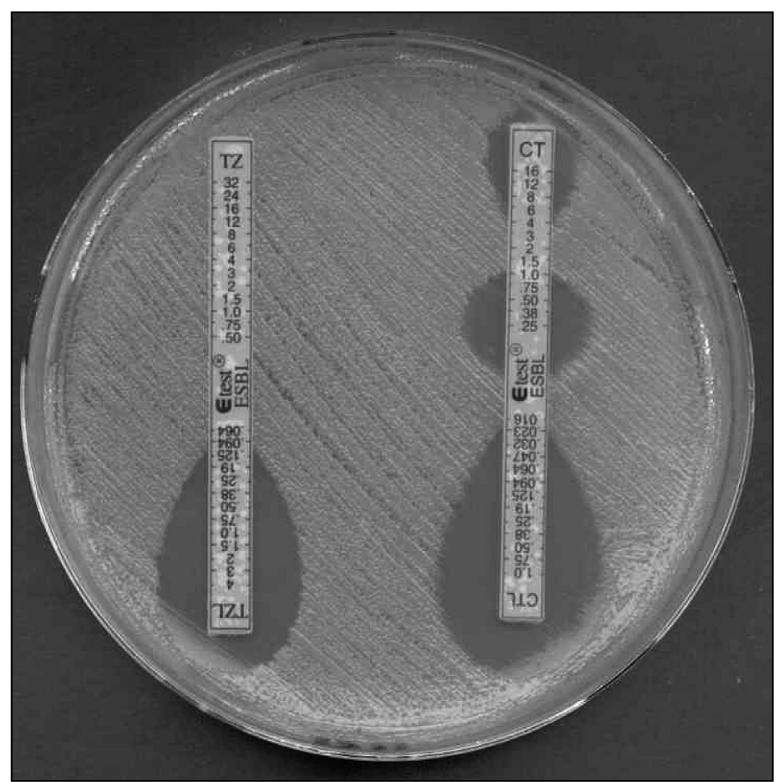

Figura VI. Conferma della produzione di ESBL mediante test di combinazione (Etest) in Klebsiella pneumoniae produttore di SHV- 12.

In alto a sinistra: TZ, ceftazidime (MIC, > $32 \mu \mathrm{g} / \mathrm{ml})$.

In basso a sinistra: TZL, ceftazidime + acido clavulanico (MIC, $0.5 \mu \mathrm{g} / \mathrm{ml})$.

In alto a destra: $\quad C T$, cefotaxime (MIC, $2 \mu \mathrm{g} / \mathrm{ml}$ ).

In basso a destra: CTL, cefotaxime + acido clavulanico (MIC, $0.094 \mu \mathrm{g} / \mathrm{ml})$. 
secuzione del test di conferma solo per E. coli, Klebsiella spp. e P. mirabilis (7), ma in linea di principio i test di conferma possono essere utilizzati anche con le altre specie di enterobatteri. Problemi interpretativi, tuttavia, possono essere incontrati con ceppi che producono $\beta$-lattamasi di tipo AmpC, quali: i) mutanti di Enterobacter spp., C. freundii, M. morganii, Providencia spp. e Serratia spp., in cui l'enzima cromosomico di tipo AmpC è prodotto in modo costitutivo (cosiddetti mutanti derepressi); ii) enterobatteri di qualunque specie (ma prevalentemente E. coli, P. mirabilis, Klebsiella spp. ed Enterobacter spp.) che hanno acquisito una $\beta$-lattamasi di tipo AmpC a codificazione plasmidica (8). Con questi ceppi, la produzione dell'enzima di tipo $\mathrm{AmpC}$, attivo sulle cefalosporine di terza generazione e non sensibile all'acido clavulanico, può mascherare la rilevazione delle ESBL nel test di conferma. In questi casi, l'impiego di una cefalosporina di quarta generazione (cefepime o cefpirome, che non sono buoni substrati per gli enzimi di tipo AmpC) appare utile per rivelare la produzione di ESBL (11).

L'iter diagnostico per lo screening e la conferma fenotipica della produzione di ESBL negli enterobatteri è riassunto in Figura VII (per E. coli, $K$. pneumoniae e P. mirabilis) e in Figura VIII (per le specie che producono $\beta$-lattamasi cromosomiche inducibili, come Enterobacter spp., M. morganii, C. freundii, Providencia spp. e Serratia spp.).

La rilevazione delle ESBL mediante sistemi automatici

Negli ultimi anni l'uso di sistemi automatici per l'identificazione e l'antibiogramma dei microrganismi si è ampiamente diffuso nei laboratori di Microbiologia Clinica. Questi sistemi rappresentano quindi spesso il primo screening per sospettare la produzione di ESBL negli enterobatteri e possono essere integrati da test dedicati alla conferma di tale meccanismo di resistenza.

In questo contesto, il ruolo e la professionalità del microbiologo appaiono cruciali sia in relazione alla scelta di sistemi automatici in grado di rilevare efficientemente le ESBL sia in relazione alla scelta dei pannelli di antibiotici più adeguati per lo screening e la conferma della produzione delle ESBL (fra le diverse opzioni di norma disponibili). Sulla base della situazione epidemiologica italiana, come già ricordato, uno screening adeguato per sospettare la presenza delle ESBL negli enterobatteri deve prevedere la possibilità di testare anche bassi livelli di MIC per almeno 2 cefalosporine (preferibilmente ceftazidime e almeno una molecola scelta fra cefotaxime, ceftriaxone e cefpodoxime). Una verifica di tale disponibilità rappresenta un elemento importante nella valutazione di un sistema automatico per l'esecuzione dell'antibiogramma.
Per quanto riguarda $i$ test di conferma della produzione di ESBL, invece, un sistema automatico dovrebbe essere in grado di valutare la sinergia dei $\beta$-lattamici con acido clavulanico, fornendo nel contempo regole di interpretazione e refertazione ad hoc. Da questo punto di vista, i principali sistemi automatici sono tutti provvisti di sistemi dedicati anche se, in realtà, pochi studi sono stati pubblicati sulla performance dei diversi sistemi e spesso questi studi non includono varianti di ESBL che rappresentano fattori di criticità (31). Pur con tali limitazioni, va sottolineato che, attualmente, i sistemi automatici hanno raggiunto elevate percentuali di sensibilità e specificità, almeno per quanto riguarda $K$. pneumoniae, E. coli e P. mirabilis (28, 30). Alcuni problemi possono invece essere riscontrati per gli enterobatteri che producono $\beta$-lattamasi di tipo AmpC (a causa della resistenza alle cefalosporine a spettro esteso nei mutanti derepressi) e per $K$. oxytoca (a causa della iperproduzione di K1, talvolta misidentificata come ESBL a causa della inibizione causata dall'acido clavulanico) (19). A questo proposito, va sottolineata la necessità di una precisa identificazione della specie batterica, in quanto tale aspetto condiziona la scelta dei test di conferma manuali e l'interpretazione dei risultati ottenuti. Sulla base di tali considerazioni, a nostro parere appare opportuno raccomandare ai laboratori che utilizzano sistemi automatici con comprovata capacità di rilevare le ESBL, l'uso di test di conferma manuali per accertare la produzione di ESBL nelle specie di enterobatteri diversi da $K$. pneumoniae, E. coli e $P$. mirabilis (seguendo le raccomandazioni riassunte in Figura VIII).

Altri caratteri fenotipici che può essere utile valutare

In aggiunta ai test che valutano la sinergia con acido clavulanico, testare la cefoxitina può essere di un certo aiuto nel confermare la produzione di ESBL: E. coli, Klebsiella spp. e P. mirabilis ESBL-positivi sono tipicamente sensibili, mentre gli isolati produttori di AmpC sono resistenti (15). Va detto che la resistenza alla cefoxitina sembra in aumento negli isolati ESBL-positivi di E. coli e Klebsiella spp. forse per la contemporanea espressione di meccanismi di efflusso o variazioni di permeabilità; di conseguenza, l'attendibilità di questo test può essere ridotta.

Caratterizzazione delle ESBL

La conferma definitiva della presenza di un gene che codifica una ESBL e la caratterizzazione del tipo e della variante enzimatica prodotta richiedono l'applicazione di metodi di biologia molecolare. In particolare, poiché le diverse varianti di ESBL differiscono tra di loro e rispetto agli enzimi da cui derivano anche solo per poche mutazioni, la conferma molecolare richiede in via conclu- 
siva il sequenziamento completo del gene. Lo studio delle caratteristiche enzimatiche delle $\beta$-lattamasi prodotte (punto isolelettrico e attività idrolitica verso i diversi substrati) è utile per orientare sul tipo di enzima ma non consente di individuare con certezza il tipo e la variante di ESBL. Il profilo di sensibilità del ceppo nei confronti dei vari $\beta$-lattamici e il comportamento nei test di conferma, consentono tuttavia di formulare delle ipotesi sul tipo di enzima più probabilmente presente.

Come deve essere fatta la refertazione con $i$ ceppi produttori di ESBL

Il rilevamento della produzione di ESBL ha un importante impatto sull'interpretazione dei risultati di sensibilità e di conseguenza sulla refertazione dell'antibiogramma. In caso di conferma con test fenotipico, secondo le indicazioni del CLSI (7) tutti i ceppi di E. coli, Klebsiella spp. e P. mirabilis produttori di ESBL vanno refertati come resistenti a tutte le penicilline, le cefalosporine (escluse le cefamicine) ed i monobattami. A nostro parere, appare prudente comportarsi nello stesso modo anche con le altre specie batteriche appartenenti alle Enterobacteriaceae.

Farmaci potenzialmente attivi nei confronti dei batteri produttori di ESBL sono considerati cefamicine, combinazioni di penicilline più inibitori delle $\beta$-lattamasi, fluorochinoloni, aminoglicosidi e carbapenemi.

Nei confronti di questi farmaci non esistono raccomandazioni internazionali che suggeriscano una modifica della refertazione.

Va sottolineato, tuttavia, che i farmaci potenzialmente attivi non hanno la stessa probabilità di successo terapeutico, specialmente nelle infezioni gravi. I risultati di studi sperimentali sugli animali e di studi clinici osservazionali di outcome indicano in maniera chiara che $i$ carbapenemi rappresentano il trattamento di scelta per le infezioni gravi causate da organismi produttori di ESBL $(10,12,32)$.

Resta invece aperta la discussione sulla possibilità di impiegare gli antibiotici non carbapenemici per le infezioni meno gravi o nel caso di ceppi con valori di MIC molto bassi (23), al fine di ridurre la pressione selettiva sui batteri Gram-negativi.

A questo riguardo, infatti, va ricordato che l'uso estensivo di imipenem è stato associato ad un aumentato riscontro di Stenotrophomonas maltophilia (naturalmente resistente ai carbapenemi) ed alla comparsa di metallo- $\beta$-lattamasi (enzimi in grado di idrolizzare $\mathrm{i}$ carbapenemi) sia negli enterobatteri che nei batteri Gramnegativi non fermentanti (17).

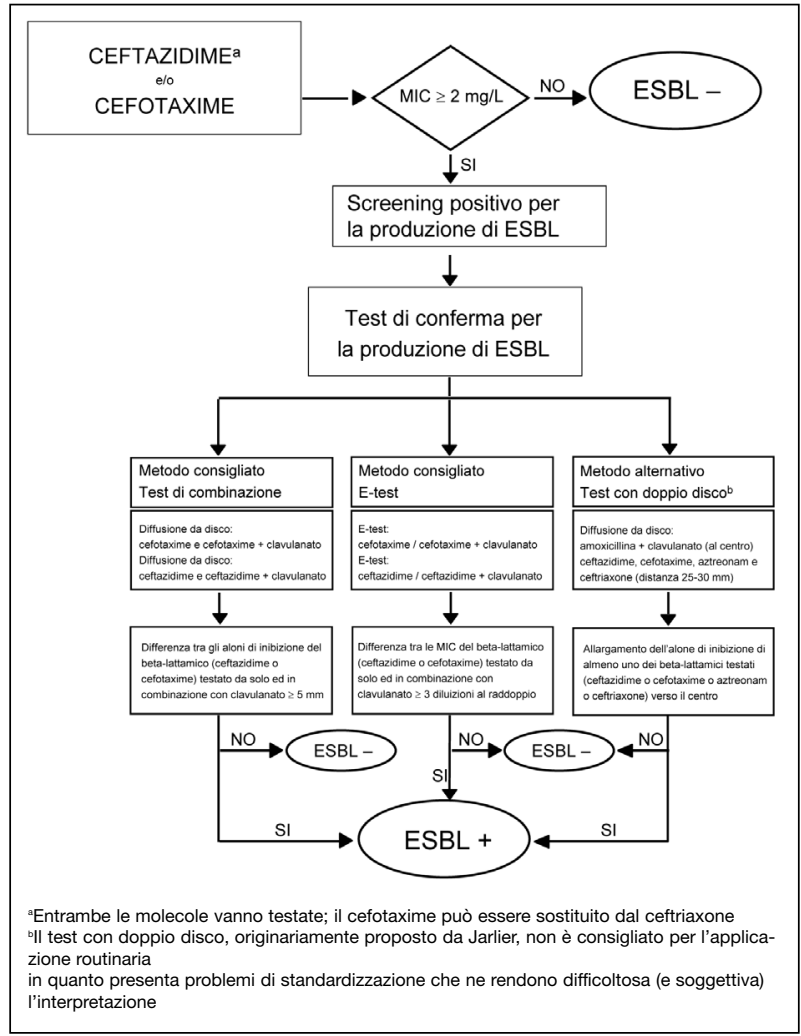

Figura VII. Iter diagnostico per lo screening e la conferma della produzione di ESBL in Escherichia coli, Klebsiella pneumoniae, Klebsiella oxytoca e Proteus mirabilis.

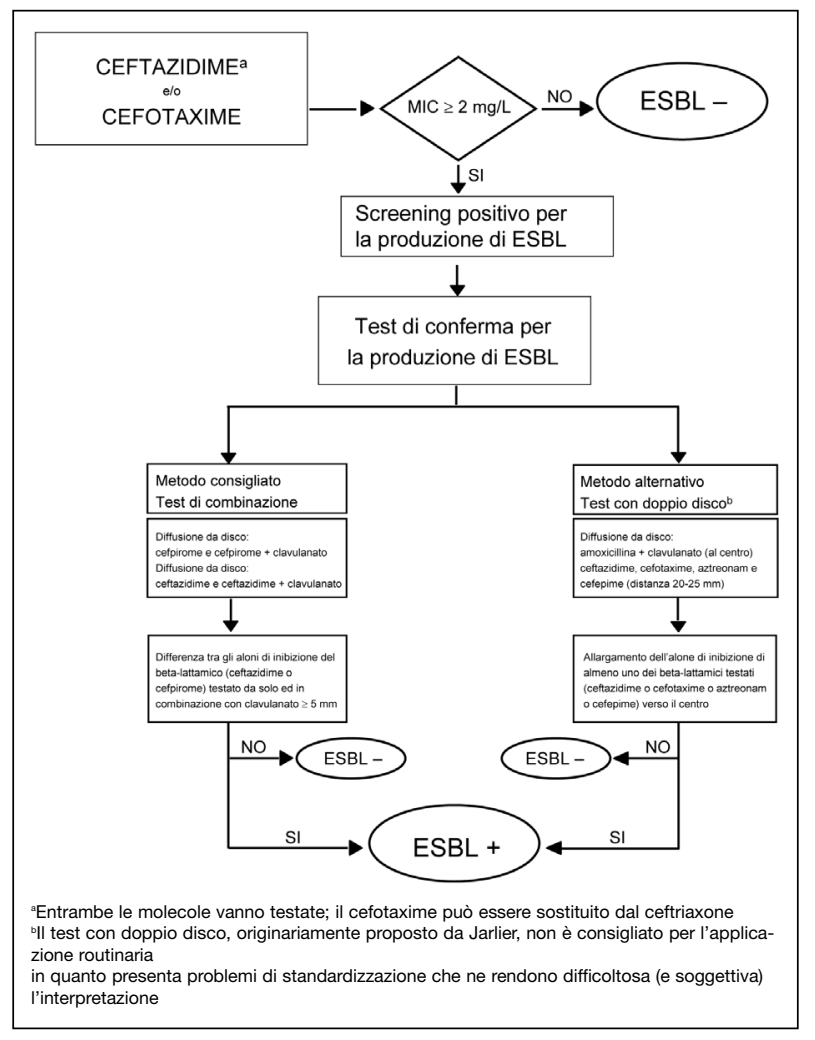

Figura VIII. Iter diagnostico per lo screening e la conferma della produzione di ESBL in Enterobacteriaceae che producono b-lattamasi cromosomiche di classe $C$ inducibili (Enterobacter spp., Citrobacter freundii, Serratia marcescens, Morganella morganii, Providencia spp.).. 


\section{BIBLIOGRAFIA}

1. Ambler RP. The structure of ß-lactamases. Phil Trans Royal Soc London, Series B Biol Sciences 1980; 289: 321-331.

2. Bonnet R. Growing group of extended-spectrum Blactamases: the CTX-M enzymes. Antimicrob Agents Chemother 2004; 48:1-14.

3. Bradford PA. Extended-spectrum $ß$-lactamases in the $21^{\text {st }}$ century: characterization, epidemiology, and detection of this important resistance threat. Clin Microbiol Rev 2001; 14: 933-951.

4. Brigante G, Luzzaro F, Perilli M, et al. Evolution of CTX-M-type ß-lactamases in isolates of Escherichia coli infecting hospital and community patients. Int $\mathrm{J}$ Antimicrob Agents 2005; 25: 157-162.

5. Bush K, Jacoby GA, and Medeiros AA. A functional classification scheme for ß-lactamases and its correlation with molecular structure. Antimicrob Agents Chemother 1995; 39: 1211-1233.

6. Canton R and Coque TM. The CTX-M ß-lactamase pandemic. Curr Opin Microbiol 2006; 9: 466-475.

7. Clinical and Laboratory Standards Institute. Performance standards for antimicrobial susceptibility testing; seventeenth informational supplement. Clinical and Laboratory Standards Institute, Wayne, PA. CLSI document 2007, M100-S17.

8. D'Andrea MM, Nucleo E, Luzzaro F, et al. CMY-16, a novel acquired AmpC-type B-lactamase of the CMY/LAT lineage in multifocal monophyletic isolates of Proteus mirabilis from northern Italy. Antimicrob Agents Chemother 2006; 50: 618-624.

9. De Vries JJ, Baas WH, van der Ploeg K, Heesink A, Degener JE, and Arends JP. Outbreak of Serratia marcescens colonization and infection traced to a healthcare worker with long-term carriage on the hands. Infect Control Hosp Epidemiol 2006; 27: 1153-1158.

10 Endimiani A, Luzzaro F, Perilli M, et al. Bacteremia due to Klebsiella pneumoniae isolates producing the TEM-52 extended-spectrum ß-lactamase: treatment outcome of patients receiving imipenem or ciprofloxacin. Clin Infect Dis 2004; 38: 243-251.

11 Health Protection Agency. Laboratory detection and reporting of bacteria with extended-spectrum B-lactamases. National Standard Method QSOP 51 Issue 2. http://www.hpa-standardmethods.org.uk/pdf_sops.asp.

12 Huang SS, Lee MH, and Leu HS. Bacteremia due to extended-spectrum $ß$-lactamase-producing Enterobacteriaceae other than Escherichia coli and Klebsiella. J Microbiol Immunol Infect 2006; 39: 496-502.

13 Jacoby GA and Munoz-Price LS. The new ß-lactamases. N Engl J Med 2005; 352: 380-391.

14 Jarlier V, Nicolas MH, Fournier G, and Philippon A. Extended-spectrum beta-lactamases conferring transferable resistance to newer beta-lactam agents in Enterobacteriaceae: hospital prevalence and susceptibility pattern. Rev Infect Dis 1988;10: 867-878.

15 Livermore DM. B-Lactamases in laboratory and clinical resistance. Clin Microbiol Rev 1995; 8: 557-584.

16 Livermore DM, Canton R, Gniadkowski M, et al. CTX-M: changing the face of ESBLs in Europe. J Antimicrob Chemother 2007; 59: 165-174.

17 Luzzaro F, Mantengoli E, Perilli M, et al. Dynamics of a nosocomial outbreak of multidrug-resistant Pseudomonas aeruginosa producing the PER-1 extended-spectrum $\beta$-lactamase. J Clin Microbiol 2001:39: 1865-1870.

18 Luzzaro F, Mezzatesta M, Mugnaioli C, et al. Trends in production of extended-spectrum $ß$-lactamases among enterobacteria of medical interest: report of the second Italian nationwide survey. J Clin Microbiol 2006; 44: 1659-1664.

19. Luzzaro F, Gesu G, Endimiani A, et al. Performance in detection and reporting B-lactam resistance phenotypes in Enterobacteriaceae: a nationwide proficiency study in Italian laboratories. Diagn Microbiol Infect Dis 2006; 55 : 311-318.

20. Mugnaioli C, Luzzaro F, De Luca F, et al. CTX-Mtype extended-spectrum ß-lactamases in Italy: molecular epidemiology of an emerging countrywide problem. Antimicrob Agents Chemother 2006; 50: 2700 2706.

21. National Nosocomial Infections Surveillance. National Nosocomial Infections Surveillance (NNIS) System Report, data summary from January 1992 through June 2004, issued October 2004. Am J Infect Control 2004; 32: 470-485.

22. Paterson DL, Ko W-C, Von Gottberg A, et al. Outcome of cephalosporin treatment for serious infections due to apparently susceptible organisms producing extended-spectrum ß-lactamases: implications for the clinical microbiology laboratory. J Clin Microbiol 2001; 39: 2206-2212.

23. Paterson DL and Bonomo RA. Extended-spectrum ßlactamases: a clinical update. Clin Microbiol Rev 2005; 18: 657-686.

24. Perilli M, Dell'Amico E, Segatore B, et al. Molecular characterization of extended-spectrum $ß$-lactamases produced by nosocomial isolates of Enterobacteriaceae from an Italian nationwide survey. J Clin Microbiol 2002; 40: 611-614.

25. Perilli M, De Santis F, Mugnaioli C, et al. Spread of Enterobacteriaceae carrying the PER-1 extendedspectrum B-lactamase gene as a chromosomal insert: a report from Italy. J Antimicrob Chemother 2007; 59: 323-324.

26. Poirel L, Menuteaeu O, Agoli N, Cattoen C, and Nordmann P. Outbreak of extended-spectrum ß-lactamase VEB-1-producing isolates of Acinetobacter baumannii in a French hospital. J Clin Microbiol 2003; 41: 3542-3547.

27. Rodriguez-Bano J, Navarro MD, Romero L, et al. Bacteremia due to extended-spectrum ß-lactamaseproducing Escherichia coli in the CTX-M era: a new clinical challenge. Clin Infect Dis 2006; 43: $1407-$ 1414.

28. Sanguinetti M, Posteraro B, Spanu $\mathrm{T}$, et al. Characterization of clinical isolates of Enterobacteriaceae from Italy by the BD Phoenix extended-spectrum ß-lactamase detection method. J Clin Microbiol 2003; 41: 1463-1468.

29. Spanu T, Luzzaro F, Perilli M, et al. Occurrence of extended-spectrum beta-lactamases in members of the family Enterobacteriaceae in Italy: implications for resistance to b-lactams and oth2002; 46: 196-202.

Francesco Luzzaro

Laboratorio di Microbiologia e Virologia Ospedale di Circolo e Fondazione Macchi Viale Borri, 57 - 21100 Varese

Tel. 0332393001 - Fax 0332260517 francesco.luzzaro@ospedale.varese.it 